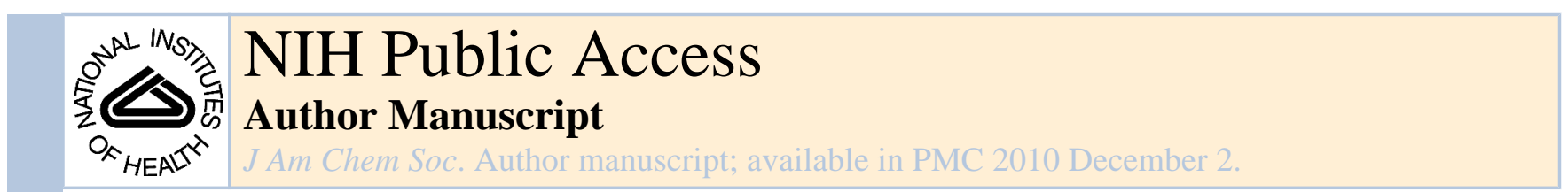

Published in final edited form as:

J Am Chem Soc. 2009 December 2; 131(47): 17052-17053. doi:10.1021/ja907479j.

\title{
An Aromatic Glaser-Hay Reaction
}

\author{
Hien-Quang Do and Olafs Daugulis ${ }^{\star}$ \\ Department of Chemistry, University of Houston, Houston, TX 77204-5003
}
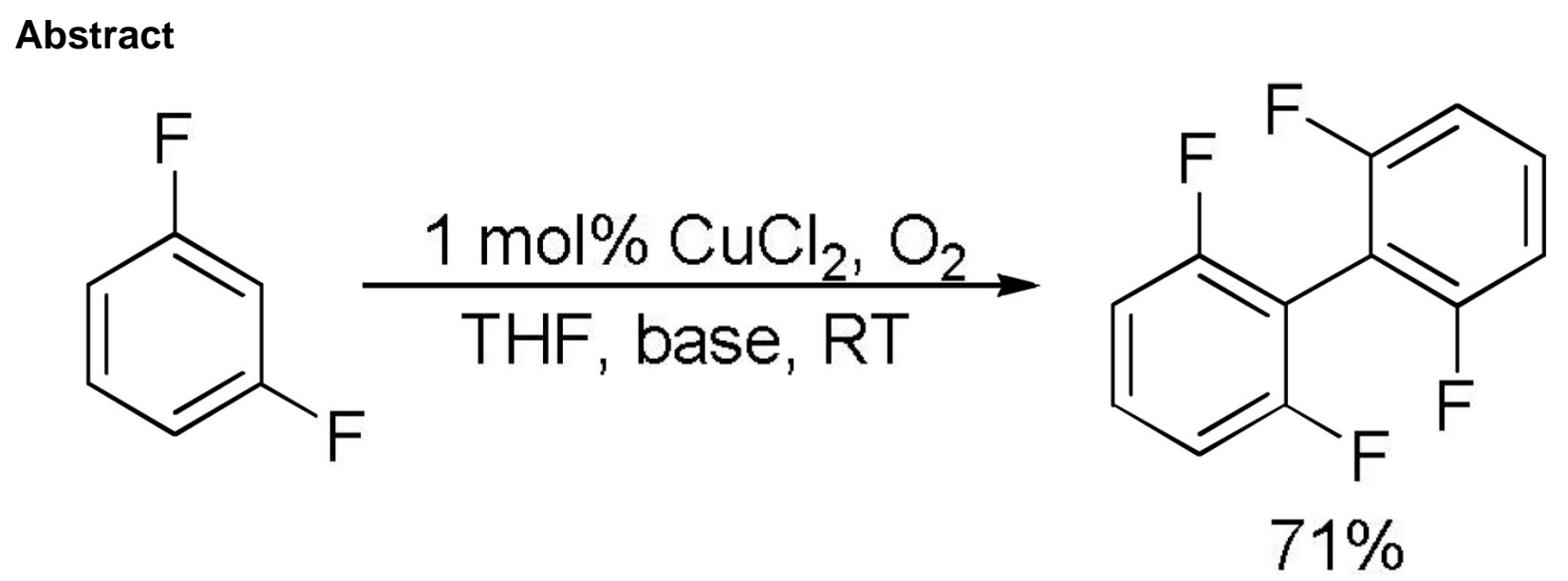

A general method for copper-catalyzed deprotonative dimerization of arenes by employing oxygen as the terminal oxidant has been developed. Electron-rich and electron-poor heterocycles as well as electron-poor arenes are reactive. The method is tolerant to functionalities such as nitro, cyano, dialkylamino, and ester groups.

Direct functionalization of C-H bonds allows to shorten synthetic schemes by allowing transformations to be carried out in fewer steps compared with traditional cross-coupling methods. ${ }^{1}$ In most cases ruthenium, rhodium, platinum, iridium, or palladium catalysts are employed. ${ }^{1 \mathrm{~h}}$ It would be advantageous to employ less exotic metals such as copper or iron for $\mathrm{C}-\mathrm{H}$ bond functionalization. Even though copper is one of the first transition metals shown to promote functionalization of $\mathrm{C}-\mathrm{H}$ bonds, ${ }^{2}$ methods that utilize copper catalysis for conversion of C-H bonds to $\mathrm{C}-\mathrm{C}$ bonds are rare. ${ }^{3}$ A notable example of such catalysis is the Glaser-Hay alkyne dimerization that has been known since 1869 (Scheme 1). ${ }^{4}$ Interestingly, oxygen is used as the terminal oxidant. Copper-catalyzed conversion of $\mathrm{C}-\mathrm{H}$ to $\mathrm{C}$-heteroatom bonds has been described, and in some cases oxygen was employed as the terminal oxidant. ${ }^{5}$ Copper-promoted 2 -arylpyridine dimerization has been recently described. ${ }^{6}$

While palladium-catalyzed arene dimerization by employing oxygen as the terminal oxidant is known, the corresponding copper-catalyzed reaction has not been reported for substrates other than phenols. ${ }^{7}$ We report here a deprotonative, copper catalyzed arene dimerization by employing oxygen as the terminal oxidant.

We have recently demonstrated that acidic $\mathrm{sp}^{2} \mathrm{C}-\mathrm{H}$ bonds can be arylated by aryl halides under copper catalysis (Scheme 2). ${ }^{8}$ The reaction involves generation of an organocopper

olafs@uh.edu.

Supporting Information Available: Experimental procedures and characterization data for new compounds. This material is available free of charge via the Internet at http://pubs.acs.org. 
intermediate followed by the reaction with aryl iodide affording a biaryl product. We speculated that under an oxygen atmosphere, the intermediate arylcopper species is expected to form biaryl and a low-valent copper complex. Regeneration of arylcopper by reaction of arylmetal with the low-valent $\mathrm{Cu}$ species would close the catalytic cycle. The initial experiments focused on the use of $t \mathrm{BuOLi}$ base that was earlier shown to be successful for copper-catalyzed deprotonative arylation. However, major amounts of phenol byproduct were formed upon reacting methoxytetrafluorobenzene with $t \mathrm{BuOLi}$ and catalytic $\mathrm{CuCl}_{2}$ under $\mathrm{O}_{2}$ atmosphere (Scheme 3). Formation of phenol byproducts has been reported in copper-catalyzed reaction of arylboronic acids with various nucleophiles under oxygen. ${ }^{9}$

Phenol can be formed either by the direct reaction of ArLi intermediate with oxygen, or by reaction of a high-valent $\mathrm{ArCu}$ with hydroxide derived from water. ${ }^{9}$ A metal that binds hydroxide removing it from the reaction mixture is required to avoid phenol formation. Less polarized C-metal bond in the intermediate should also decrease the reactivity of arylmetal with oxygen. Magnesium $t$-butoxide was inefficient and a stronger base was required. Fortunately, hindered zinc and magnesium amide bases that have been extensively investigated by Knochel worked well. ${ }^{10}$ Exact composition of the base needs to be optimized for each substrate. Synthesis of bases used in dimerizations is presented in Scheme 4. Best results are obtained by employing tetramethylpiperidides.

The results of the dimerization reactions are shown in Table 1. Reactions are run in THF solvent under oxygen atmosphere at $0-50{ }^{\circ} \mathrm{C}$, typically at RT, and $1-3 \mathrm{~mol} \%$ of $\mathrm{CuCl}_{2}$ catalyst is employed. Electron-rich heterocycles such as thiazole, benzofuran, 2-chlorothiophene, $\mathrm{N}$ butylimidazole and triazole are dimerized in good yields (entries 1-5). Electron-poor heterocycles 2-methoxypyrazine and 3,5-dichloropyridine were also dimerized successfully (entries 6 and 7). Polyfluorinated arenes are reactive and tetrafluoroanisole was dimerized in $91 \%$ yield (entry 8). 1,3-Difluorobenzene afforded tetrafluorobiphenyl in 71\% yield (entry 10). The reaction is tolerant to functional groups such as amino (entry 9), nitro (entry 11), cyano (entry 12), and ester (entry 13). For dimerization of 2-chlorothiophene and tetrafluoroanisole cheaper dicyclohexylamide bases can be employed. In other cases, tetramethylpiperidides afford better results.

Control experiments were run to determine if trace of another transition metal catalyzes the dimerization (Scheme 5). ${ }^{11}$ With reagent grade or ultra-pure $\mathrm{CuCl}_{2}$ similar results were obtained showing that reactivity by contaminants is unlikely. If copper salt was omitted, no product was obtained.

In conclusion, we have developed a general method for copper-catalyzed, deprotonative dimerization of arenes by employing oxygen as the terminal oxidant. Electron-rich and electron-poor heterocycles as well as electron-poor arenes are reactive. The method is tolerant to functionalities such as nitro, cyano, amino, and ester groups.

\section{Supplementary Material}

Refer to Web version on PubMed Central for supplementary material.

\section{Acknowledgments}

We thank the Welch Foundation (Grant No. E-1571), National Institute of General Medical Sciences (Grant No. R01GM077635), A. P. Sloan Foundation, Camille and Henry Dreyfus Foundation, and Norman Hackerman Advanced Research Program for supporting this research. 


\section{REFERENCES}

1. (a) Lewis JC, Bergman RG, Ellman JA. Acc. Chem. Res 2008;41:1013. [PubMed: 18616300] (b) Campeau L-C, Fagnou K. Chem. Commun 2006:1253. (c) Chen X, Engle KM, Wang D-H, Yu J-Q. Angew. Chem., Int. Ed 2009;48:5094. (d) Seregin IV, Gevorgyan V. Chem. Soc. Rev 2007;36:1173. [PubMed: 17576484] (e) Dick AR, Sanford MS. Tetrahedron 2006;62:2439. (f) Daugulis O, Do H-Q, Shabashov D. Acc. Chem. Res 2009;42:1074. [PubMed: 19552413] (g) Alberico D, Scott ME, Lautens M. Chem. Rev 2007;107:174. [PubMed: 17212475]

2. Steinkopf W, Leitsmann R, Hofmann KH. Liebigs Ann. Chem 1941;546:180.

3. (a) Phipps RJ, Gaunt MJ. Science 2009;323:1593. [PubMed: 19299616] (b) Yoshizumi T, Tsurugi H, Satoh T, Miura M. Tetrahedron Lett 2008;49:1598. (c) Besselièvre F, Piguel S, Mahuteau-Betzer F, Grierson DS. Org. Lett 2008;10:4029. [PubMed: 18720988] (d) Ackermann L, Potukuchi HK, Landsberg D, Vicente R. Org. Lett 2008;10:3081. [PubMed: 18549230] (e) Li Z, Li C-J. J. Am. Chem. Soc 2005;127:6968. [PubMed: 15884937] (f) Yotphan S, Bergman RG, Ellman JA. Org. Lett 2009;11:1511. [PubMed: 19260648]

4. (a) Glaser C. Ber. Dtsch. Chem. Ges 1869;2:422. (b) Hay AS. J. Org. Chem 1962;27:3320.

5. (a) Basle O, Li C-J. Chem. Commun 2009:4124. (b) Chen X, Hao X-S, Goodhue CE, Yu J-Q. J. Am. Chem. Soc 2006;128:6790. [PubMed: 16719450] (c) Hamada T, Ye X, Stahl SS. J. Am. Chem. Soc 2008;130:833. [PubMed: 18166058] (d) Monguchi D, Fujiwara T, Furukawa H, Mori A. Org. Lett 2009;11:1607. [PubMed: 19254040] (e) Brasche G, Buchwald SL. Angew. Chem., Int. Ed 2008;47:1932.

6. Chen X, Dobereiner G, Hao X-S, Giri R, Maugel N, Yu J-Q. Tetrahedron 2009;65:3085.

7. Pd-catalyzed arene intermolecular homo/heterodimerization: (a) Okamoto M, Yamaji T. Chem. Lett 2001:212. (b) Brasche G, García-Fortanet J, Buchwald SL. Org. Lett 2008;10:2207. [PubMed: 18465866] (c) Hagelin H, Oslob JD, Åkermark B. Chem.-Eur. J 1999;5:2413. Grignard homodimerization under $\mathrm{O}_{2}$ : (d) Cahiez G, Moyeux A, Buendia J, Duplais C. J. Am. Chem. Soc 2007;129:13788. [PubMed: 17944469] (e) Maji MS, Pfeifer T, Studer A. Angew. Chem., Int. Ed 2008;47:9547. Phenol dimerization under $\mathrm{Cu} / \mathrm{O}_{2}$ : (f) Sakamoto T, Yonehara H, Pac C. J. Org. Chem 1994;59:6859. Enolate homocoupling: (g) DeMartino MP, Chen K, Baran PS. J. Am. Chem. Soc 2008;130:11546. [PubMed: 18680297]

8. (a) Do H-Q, Daugulis O. J. Am. Chem. Soc 2007;129:12404. [PubMed: 17887762] (b) Do H-Q, Daugulis O. J. Am. Chem. Soc 2008;130:1128. [PubMed: 18181627] (c) Do H-Q, Khan RMK, Daugulis O. J. Am. Chem. Soc 2008;130:15185. [PubMed: 18855471]

9. (a) King AE, Brunold TC, Stahl SS. J. Am. Chem Soc 2009;131:5044. [PubMed: 19309072] (b) Lam PYS, Bonne D, Vincent G, Clark CG, Combs AP. Tetrahedron Lett 2003;44:1691. (c) Demir AS, Reis Ö, Emruallahoglu M. J. Org. Chem 2003;68:10130. [PubMed: 14682710]

10. (a) Mosrin M, Knochel P. Org. Lett 2009;11:1837. [PubMed: 19317432] (b) Dubbaka SR, Kienle M, Mayr H, Knochel P. Angew. Chem., Int. Ed 2007;46:9093. (c) Wunderlich SH, Knochel P. Angew. Chem., Int. Ed 2007;46:7685.

11. Buchwald SL, Bolm C. Angew. Chem., Int. Ed 2009;48:5586. 


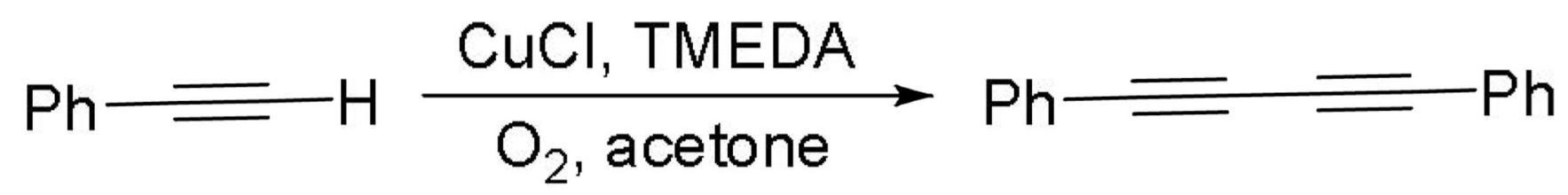

Scheme 1.

Glaser-Hay reaction 


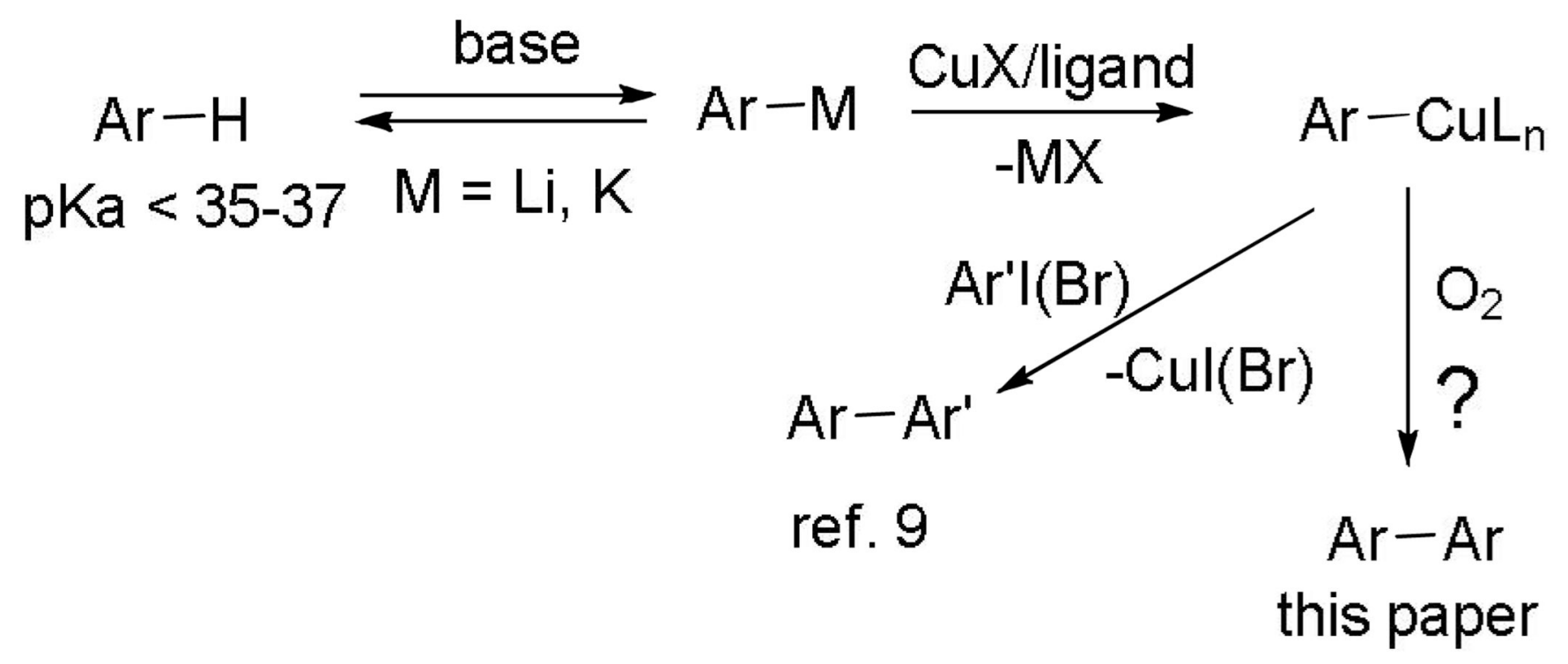

Scheme 2.

Copper-catalyzed arylation of arene $\mathrm{C}-\mathrm{H}$ bonds 
<smiles>COc1c(F)c(F)c(-c2c(F)c(F)c(OC)c(F)c2F)c(F)c1F</smiles><smiles>COc1c(F)c(F)c(O)c(F)c1F</smiles>

Scheme 3.

Phenol byproduct formation 
Base 1 iPrMgCl${ }^{\star} \mathrm{LiCl}+$ tetramethylpiperidine (1:1.1)

Base 2 Base $1+\mathrm{ZnCl}_{2}(1: 0.25)$

Base 3 Dicyclohexylamine + iPrMgCl${ }^{*} \mathrm{LiCl}+\mathrm{ZnCl}_{2}(1.1: 1: 0.25)$

Base 4 Base $1+\mathrm{ZnCl}_{2}(1: 0.5)$

Scheme 4.

Bases employed in dimerization 
<smiles>Brn1cnnc1</smiles>
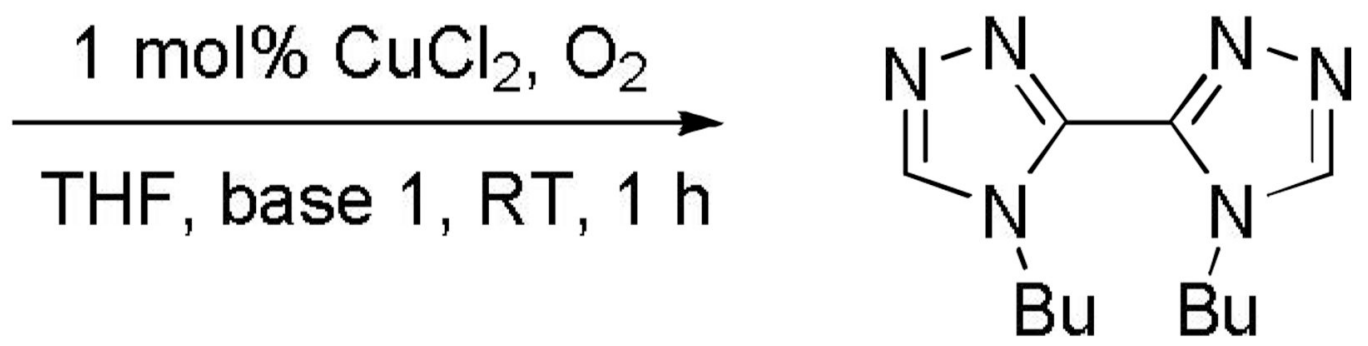

$98 \% \mathrm{CuCl}_{2}$ (reagent grade), $73 \%$ isolated yield $99.999 \% \mathrm{CuCl}_{2}$ (ultra pure), $77 \%$ isolated yield no $\mathrm{CuCl}_{2},<2 \%$ conversion

Scheme 5.

Control Experiments 
Table 1

Arene Dimerization $^{a}$

$$
\mathrm{Ar}-\mathrm{H} \frac{1-3 \mathrm{~mol} \% \mathrm{CuCl}_{2}, \mathrm{O}_{2}}{\mathrm{THF}, \text { base, } 0-50{ }^{\circ} \mathrm{C}} \mathrm{Ar}-\mathrm{Ar}
$$

EntryAr-H, base

Product

1 Thiazole, base 4

2 Benzofuran, base 1$$
\left.||_{n}^{S}\right\rangle
$$
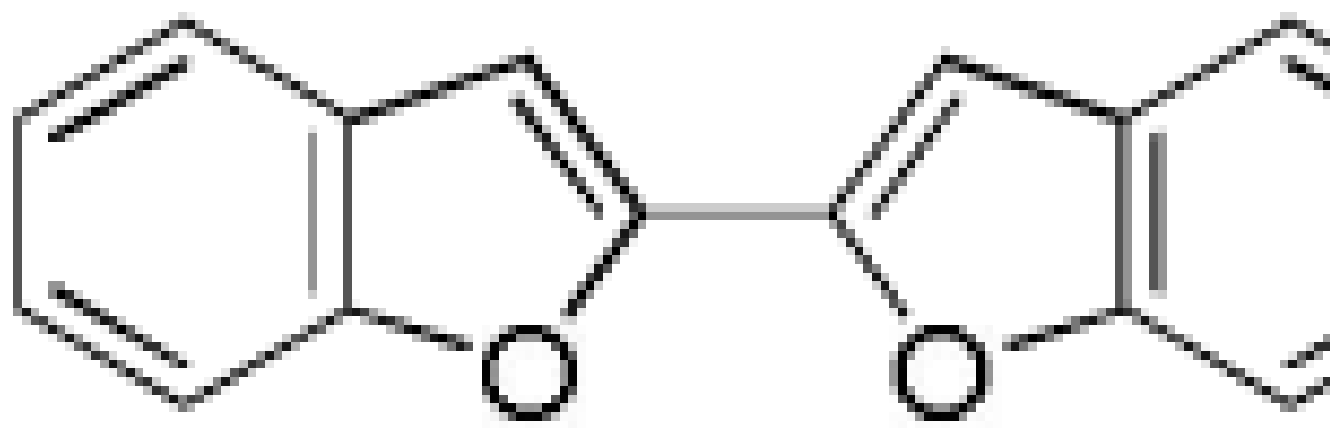

3 2-Chlorothiophene, base 3

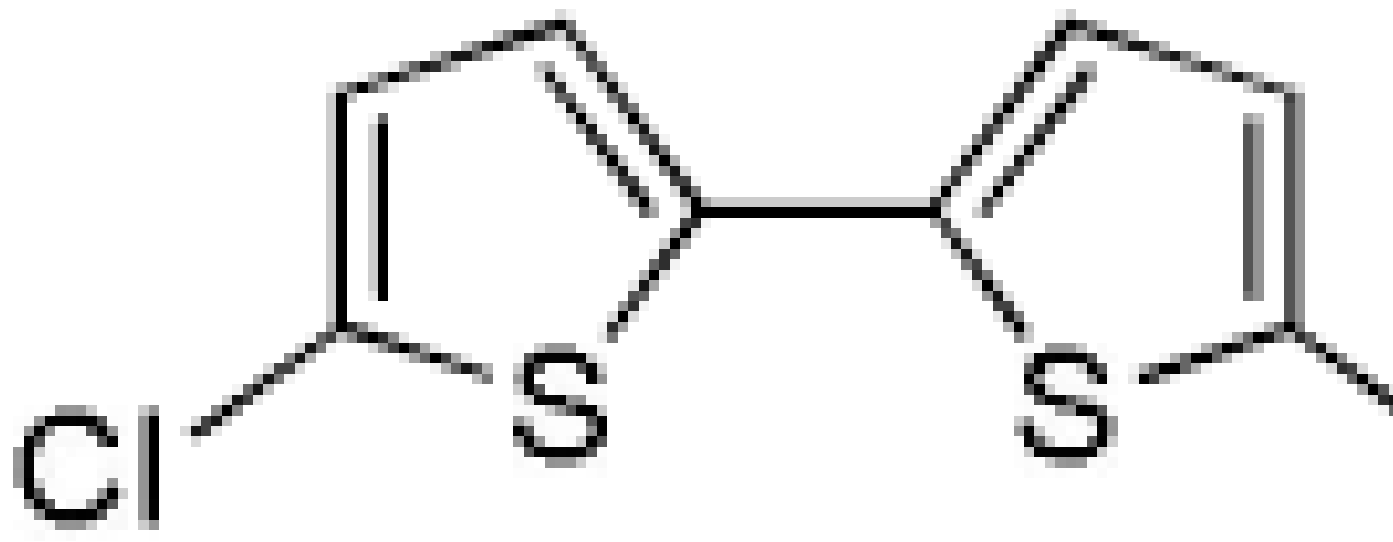




$$
\mathrm{Ar}-\mathrm{H} \underset{\mathrm{THF} \text {, base, } 0-50{ }^{\circ} \mathrm{C}}{\stackrel{1-3 \mathrm{~mol} \% \mathrm{CuCl}_{2}, \mathrm{O}_{2}}{\longrightarrow}} \mathrm{Ar}-\mathrm{Ar}
$$

$4 \mathrm{~N}$-Butylimidazole, base 1
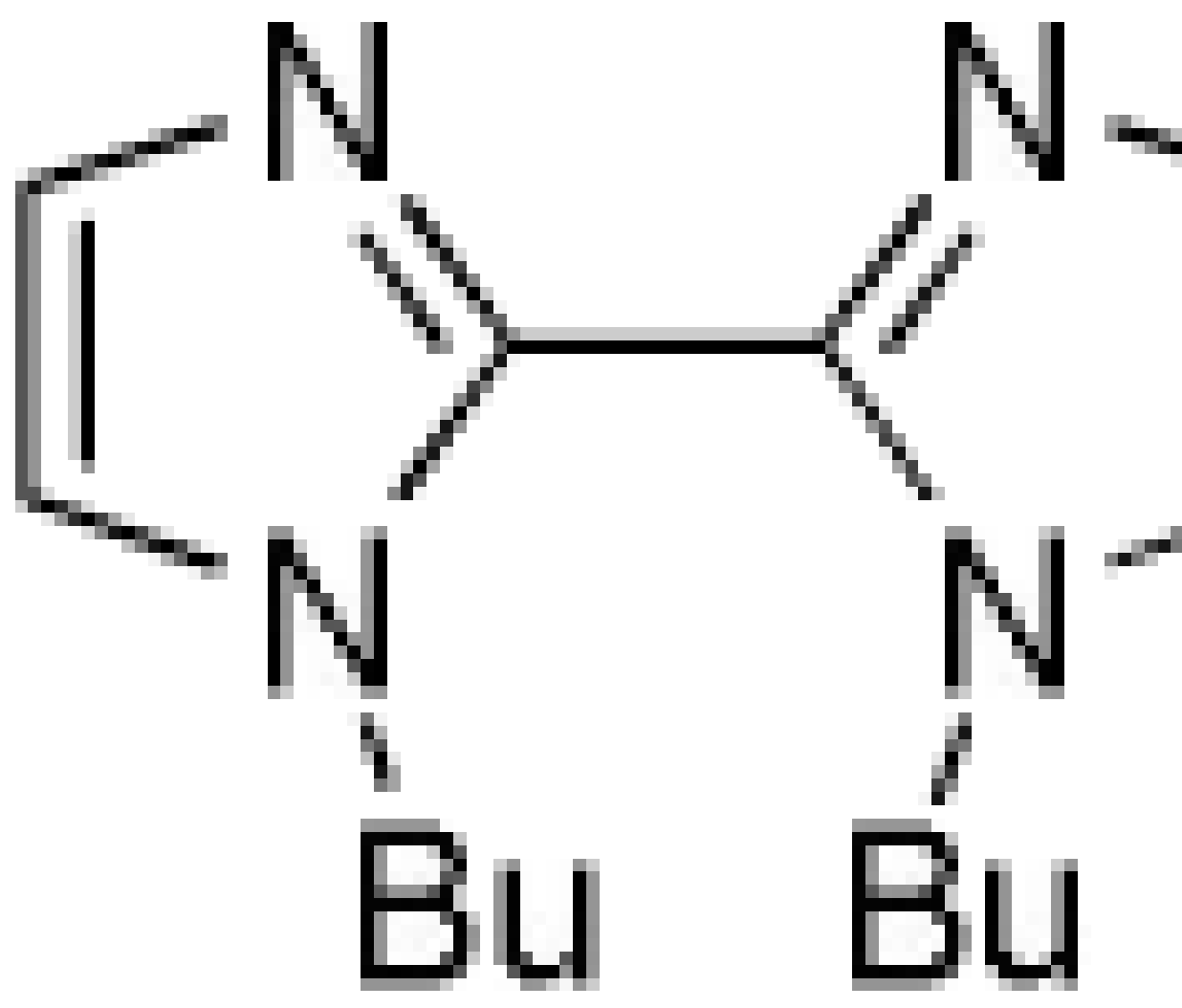

$5 \quad N$-Butyltriazole, base 1

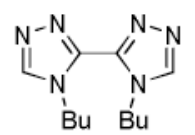




$$
\mathrm{Ar}-\mathrm{H} \frac{1-3 \mathrm{~mol}^{2} \mathrm{CuCl}_{2}, \mathrm{O}_{2}}{\mathrm{THF}, \text { base, } 0-50{ }^{\circ} \mathrm{C}} \mathrm{Ar}-\mathrm{Ar}
$$

6 Methoxypyrazine, base $1+$ base 4
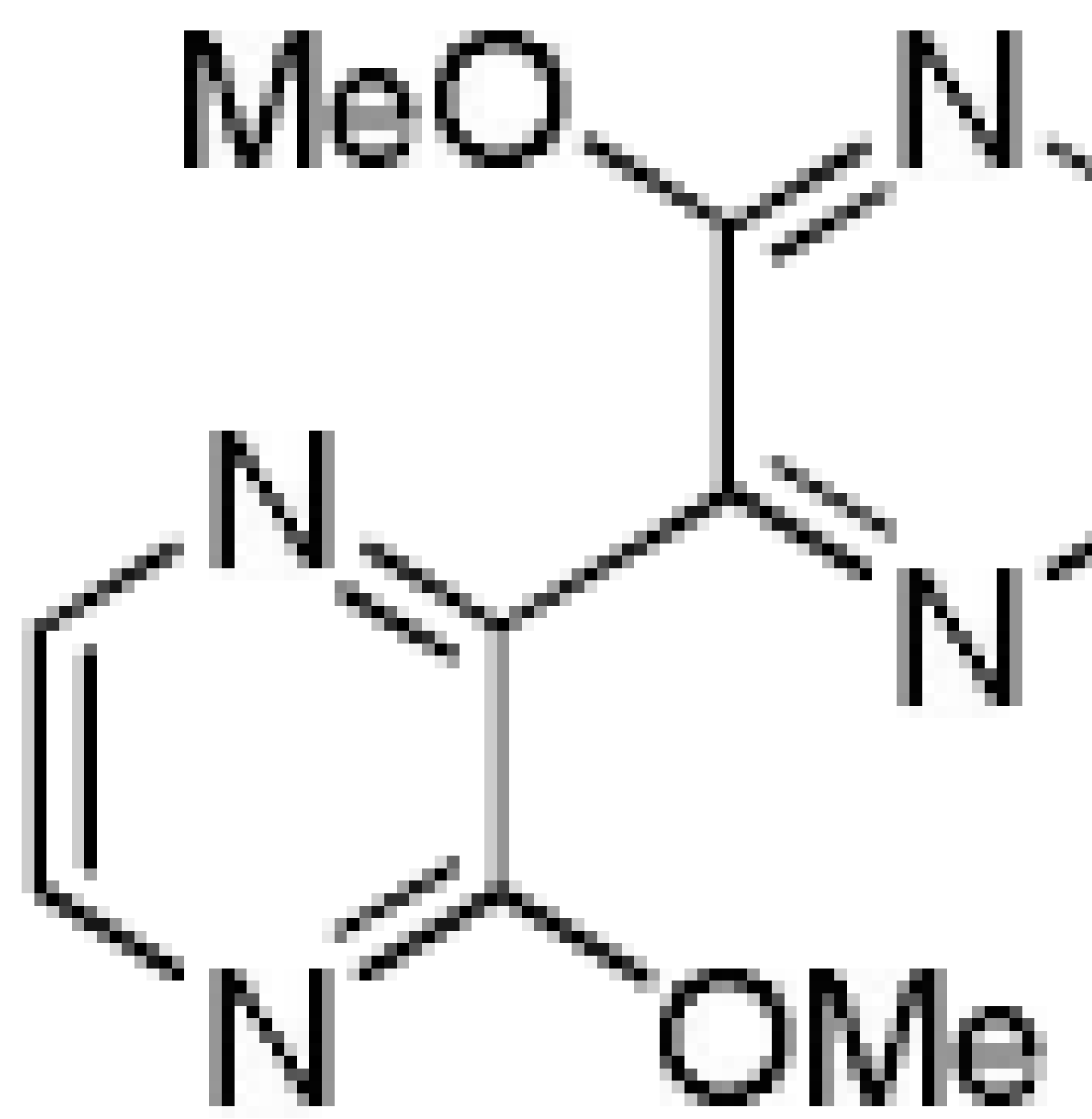

7 3,5-Dichloropyridine, base 2

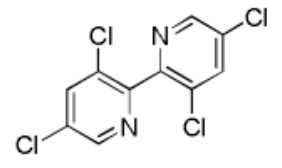




$$
\mathrm{Ar}-\mathrm{H} \frac{1-3 \mathrm{~mol}^{2} \mathrm{CuCl}_{2}, \mathrm{O}_{2}}{\mathrm{THF}, \text { base, } 0-50{ }^{\circ} \mathrm{C}} \mathrm{Ar}-\mathrm{Ar}
$$

EntryAr-H, base

Product

8 Tetrafluoroanisole base 3

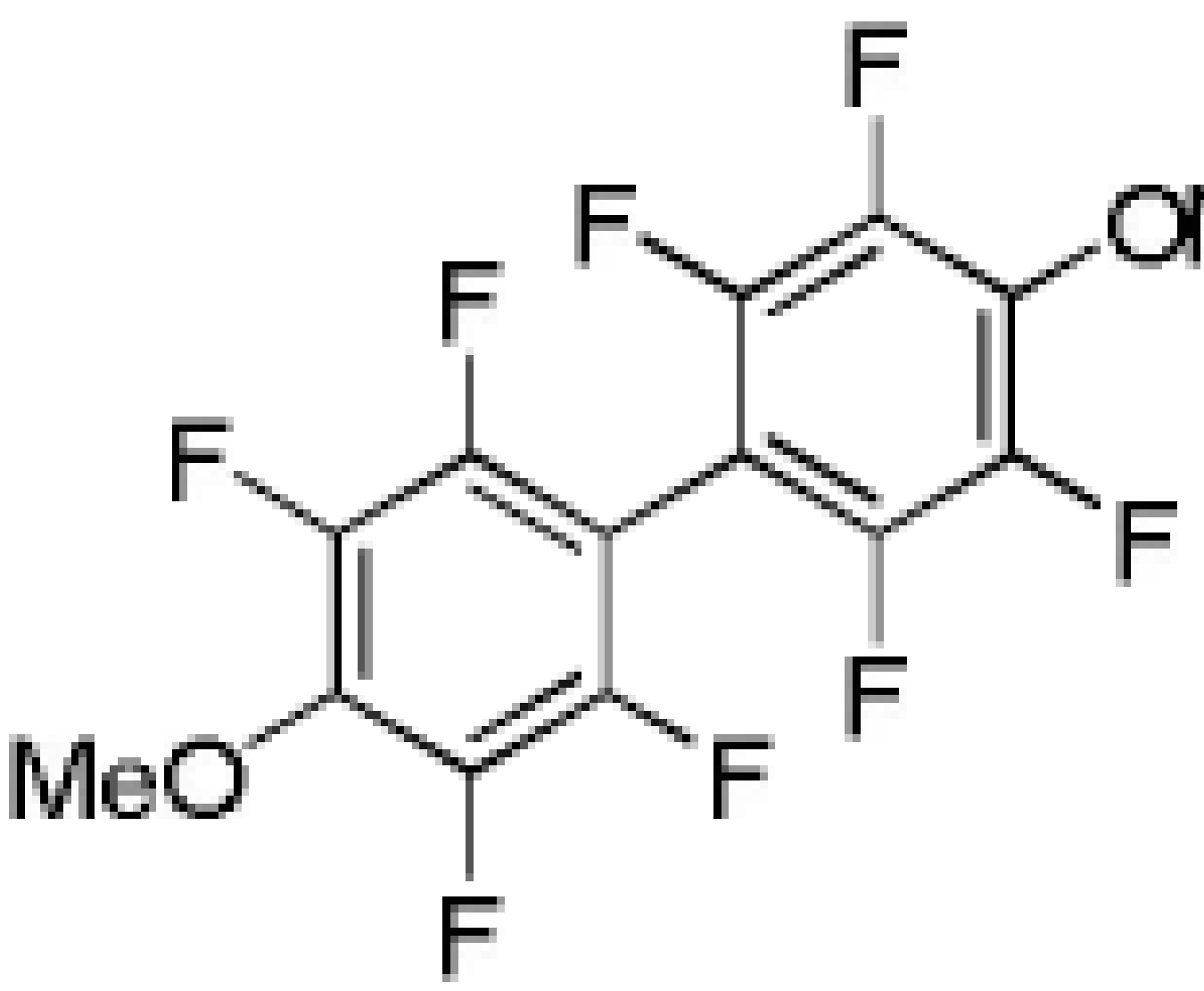




$$
\mathrm{Ar}-\mathrm{H} \underset{\mathrm{THF}, \text { base, } 0-50^{\circ} \mathrm{C}}{\stackrel{1-3 \mathrm{~mol} \% \mathrm{CuCl}_{2}, \mathrm{O}_{2}}{\longrightarrow}} \mathrm{Ar}-\mathrm{Ar}
$$

EntryAr-H, base

Product

9 Tetrafluoro-N,N-dimethylaniline, base 4

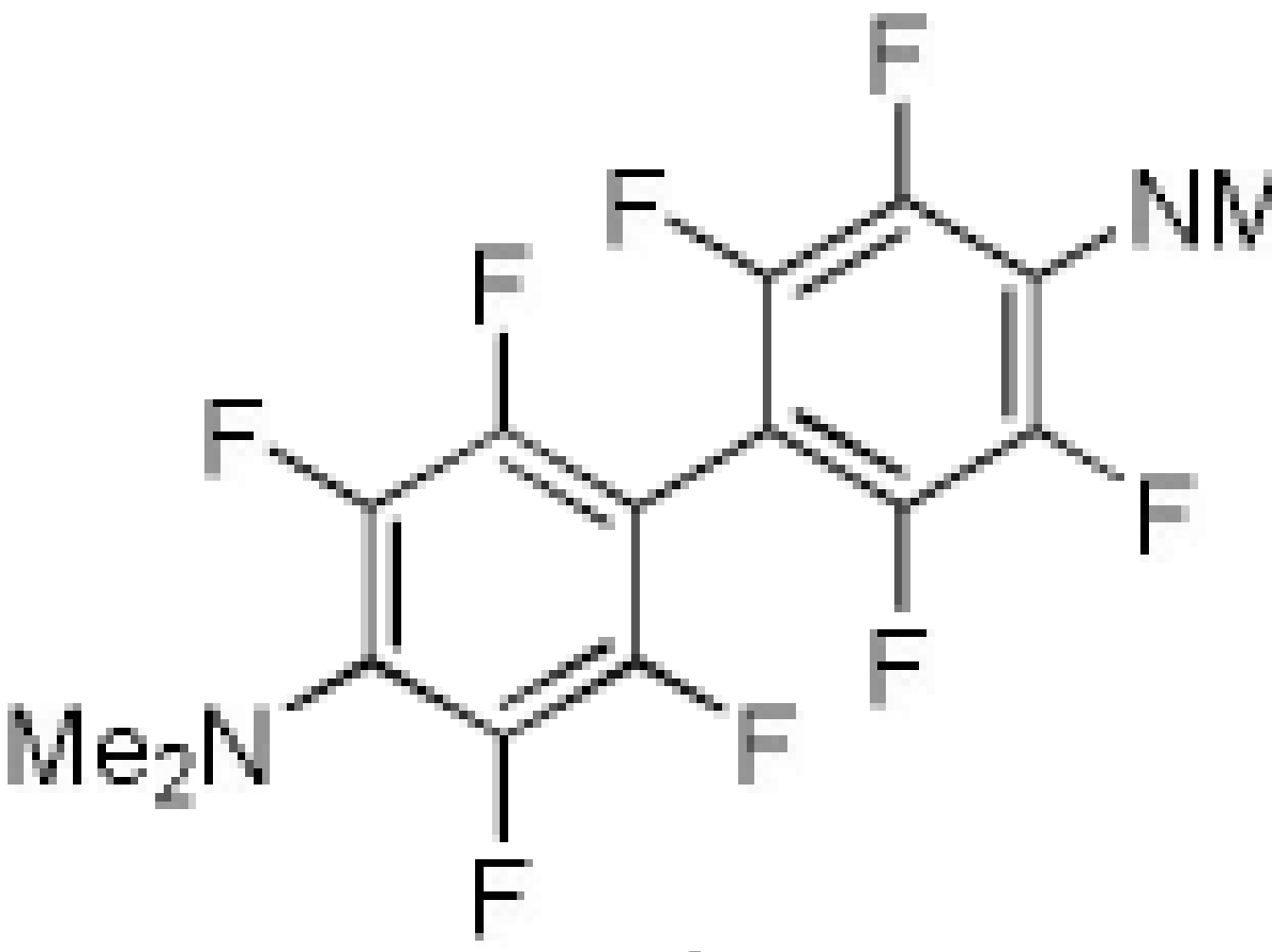

10 1,3-Difluorobenzene, base 2

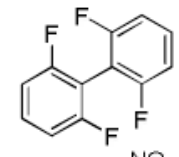

11 2,4-Difluoronitrobenzene, base 4

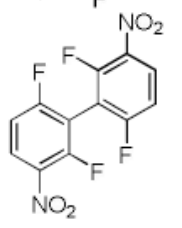




$$
\mathrm{Ar}-\mathrm{H} \frac{1-3 \mathrm{~mol}^{2} \mathrm{CuCl}_{2}, \mathrm{O}_{2}}{\mathrm{THF}, \text { base, } 0-50{ }^{\circ} \mathrm{C}} \mathrm{Ar}-\mathrm{Ar}
$$

EntryAr-H, base

Product

12 3,5-Difluorobenzonitrile, base 4 + base 1

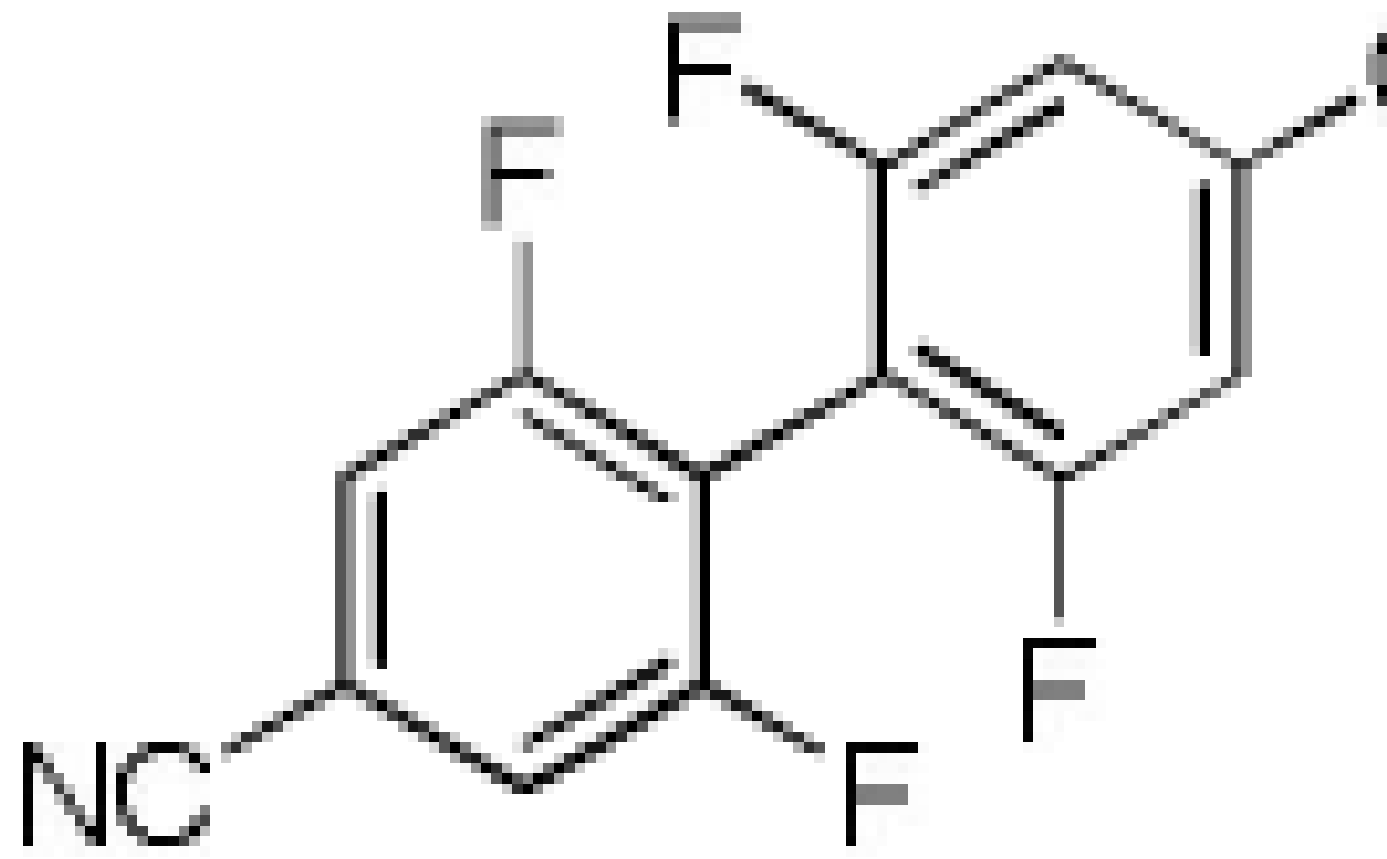




$$
\mathrm{Ar}-\mathrm{H} \underset{\mathrm{THF}, \text { base, } 0-50{ }^{\circ} \mathrm{C}}{\stackrel{1-3 \mathrm{~mol} \% \mathrm{CuCl}_{2}, \mathrm{O}_{2}}{\longrightarrow}} \mathrm{Ar}-\mathrm{Ar}
$$

13 Ethyl 3,4-difluorobenzoate, base 1

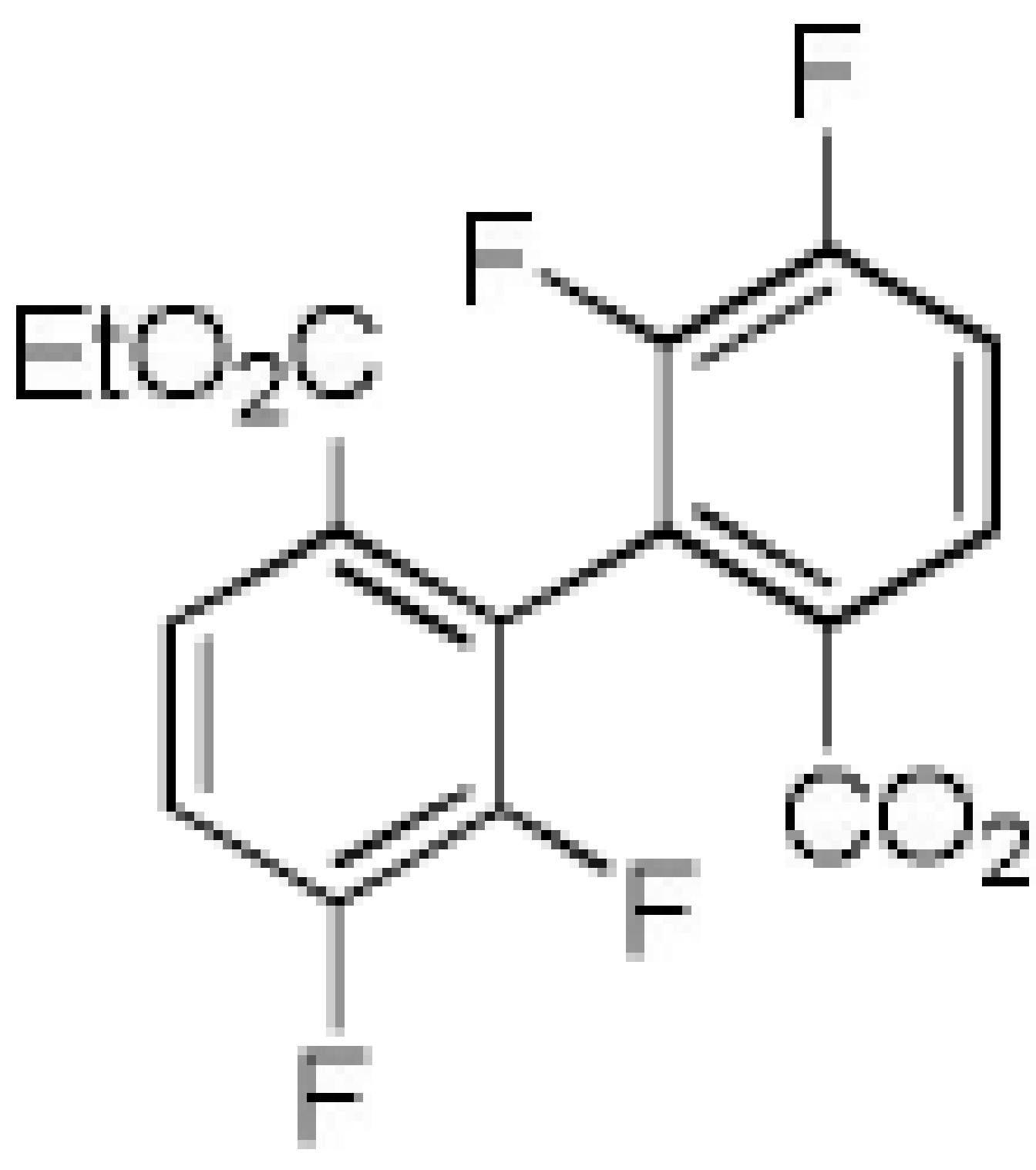

${ }^{a}$ Substrate (1 equiv), base (1.2-1.5 equiv). Yields are isolated yields. See Supporting Information for details. 